Article

\title{
Regioselective Synthesis of Procyanidin B6, A 4-6-Condensed (+)-Catechin Dimer, by Intramolecular Condensation
}

\author{
Yusuke Higashino ${ }^{1}$, Taisuke Okamoto ${ }^{1}$, Kazuki Mori ${ }^{1}$, Takashi Kawasaki ${ }^{2}$, \\ Masahiro Hamada ${ }^{3}$, Noriyuki Nakajima ${ }^{3}$ and Akiko Saito ${ }^{1, *}$ \\ 1 Graduate School of Engineering, Osaka Electro-Communication University (OECU), 18-8 Hatsu-cho, \\ Neyagawa-shi, Osaka 572-8530, Japan; de15a002@oecu.jp (Y.H.); me12a004@osakac.info (T.O.); \\ me14a009@oecu.jp (K.M.) \\ 2 College of Pharmaceutical Sciences, Ritsumeikan University, 1-1-1 Nojihigashi, Kusatsu, \\ Shiga 525-8577, Japan; kawa0227@fc.ritsumei.ac.jp \\ 3 Department of Pharmaceutical Engineering, Faculty of Engineering, Toyama Prefectural University (TPU), \\ 5180, Kurokawa, Imizu, Toyama 939-0398, Japan; hamada@pu-toyama.ac.jp (M.H.); \\ nori@pu-toyama.ac.jp (N.N.) \\ * Correspondence: a-saito@osakac.ac.jp; Tel.: +81-72-824-1131
}

Received: 9 January 2018; Accepted: 16 January 2018; Published: 18 January 2018

\begin{abstract}
Proanthocyanidins, also known as condensed tannins or oligomeric flavonoids, are found in many edible plants and exhibit interesting biological activities. Herein, we report a new, simple method for the stereoselective synthesis of procyanidin B6, a (+)-catechin-(4-6)-(+)-catechin dimer, by Lewis acid-catalyzed intramolecular condensation. The 5-O-t-butyldimethylsilyl (TBDMS) group of 5,7,3' $4^{\prime}$-tetra-O-TBDMS-(+)-catechin was regioselectively removed using trifluoroacetic acid, leading to the "regio-controlled" synthesis of procyanidin B6. The 5-hydroxyl group of the 7,3', $4^{\prime}$-tri-O-TBDMS-(+)-catechin nucleophile and the 3-hydroxyl group of $5,7,3^{\prime}, 4^{\prime}$-tetra-O-benzylated-(+)-catechin electrophile were connected with an azelaic acid. The subsequent $\mathrm{SnCl}_{4}$-catalyzed intramolecular condensation proceeded smoothly to give the 4-6-condensed catechin dimer. This is the first report on the complete regioselective synthesis of a 4-6-connected oligomer without modifying the 8-position.
\end{abstract}

Keywords: condensed tannin; proanthocyanidins; intramolecular condensation; procyanidin B6

\section{Introduction}

Proanthocyanidins are a class of polyphenols and are generally referred to as condensed tannins or oligomeric flavonoids. They have attracted a great deal of attention because of their strong antioxidant activity and wide range of interesting biological functions [1,2]. However, proanthocyanidins are often obtained as a mixture of various analogues from plants. Thus, despite their simple structure as the derivatives of flavan-3-ols, the purification of each compound remains difficult. Flavan-3-ols such as catechin and epicatechin are one of the most well-known groups of biologically active polyphenols. The oligomers composed of these catechins and epicatechin are known as highly functional polyphenols contained in familiar foods such as cacao [3,4]. There are many reports related to other biological activities, such as antifungal [5,6], antiviral [5,7], anti-inflammatory [8], anticancer [3,9], and treating heart diseases [10], among others. Flavan-3-ol derivatives including proanthocyanidins are also converted to various structurally complex secondary polyphenols through chemical reactions that occur during food processing such as drying [11], frozen storage [12], and acidic treatment [13]. These complex and diverse forms make the isolation, structural determination, and evaluation of the biological activities of each compound much more difficult. Therefore, securing 
sufficient amount of proanthocyanidin as a pure compound for experiment is also important for elucidating the function of proanthocyanidin. A synthetic route to oligomeric catechin and epicatechin derivatives has been developed by Kozikowski et al. [14-16] and subsequently by other research groups [17-24]. Additionally, our group has developed and reported simple and stereoselective methods to synthesize 4-8 condensed procyanidin oligomers (constituted by flavan-3-ols having two hydroxyl groups on B and E rings) [25-38] and prodelphinidin oligomers (constituted by flavan-3-ols having, three hydroxyl groups on B and/or E rings) [39] and then studied their biological activities. The key step of the synthesis of proanthocyanidins is the coupling reaction between the nucleophile and electrophile using a Lewis acid such as $\mathrm{TiCl}_{4}, \mathrm{SnCl}_{4}$, or trimethylsilyl triflate (TMSOTf) as an activator. As shown in Figure 1, the two dimers of (+)-catechin, 4-6-condensed procyanidin B6 (1) and 4-8-condensed procyanidin B3 (2), are isomers with different bonding positions. The abundance of the 4-6-linked oligomer is comparatively low. In particular, the biological activities of various 4-6-condensed oligomers are yet to be studied in detail due to the unavailability of these compounds. Most studies on the synthesis of proanthocyanidins focus on 4-8-linked oligomers as target compounds, and only a few of them address the stereoselective synthesis of 4-6-linked oligomers. The cool synthesis of the 4-6 catechin dimer, procyanidin B6 (1), has been previously achieved by combining the 8-halo-capping strategy [24].

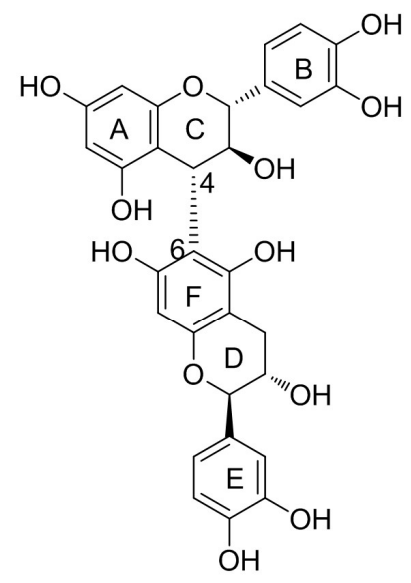

Procyanidin B6 (1)

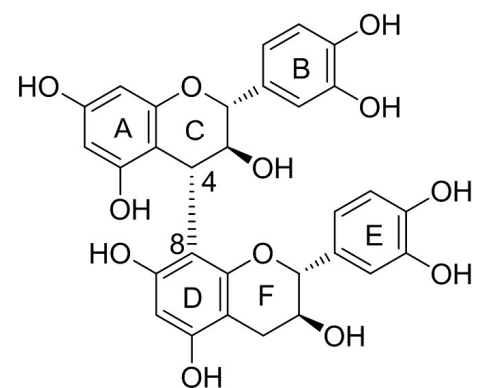

Procyanidin B3 (2)

Figure 1. Structures of procyanidin B6 (1) and procyanidin B3 (2).

Due to increasing interest toward the structure-activity-relationship studies (SAR) of proanthocyanidins, developing new and simple methods for the synthesis of various types of proanthocyanidins has become increasingly important. Therefore, we focused on developing a very simple approach to the selective synthesis of such compounds. Herein, we report on an easy, applicable, and reliable method for the stereoselective synthesis of procyanidin B6 (1) via an intramolecular condensation reaction without modifying the 8-position.

\section{Results and Discussion}

Previously, we developed a Lewis acid-catalyzed "intramolecular" one-to-one coupling approach that allows for the stereoselective synthesis of 4-8-condensed (+)-catechin dimers (4, 6: Scheme 1) [28,31]. The method includes the connection of flavan-3-ol-derived nucleophile and electrophile via a diester linker and the subsequent condensation reaction by "intramolecular" coupling. Furthermore, the stereochemistry of the coupling product was found to depend on the length of the linker. Despite the fact that the 3,4-cis structure is not preferentially obtained in intermolecular reactions, the intramolecular TMSOTf-catalyzed coupling of azelaic acid (C9 dicarboxylic acid) diester (3) afforded 3,4-trans product 4, and the coupling of glutaryl (C3 dicarboxylic acid derivative) diester 
(5) gave 3,4-cis product 6 [28] (Scheme 1). Therefore, this intramolecular coupling method could potentially solve the difficulties surrounding the stereoselective synthesis of proanthocyanidins such as 3,4-cis oligomers. Additionally, it could be possible that the structure of the intramolecular coupling product is changed depending on the position in which the diester linker is introduced. Following these assumptions, we attempted to introduce a diester linker regioselectively to another position by selectively deprotecting one of the four protecting groups of the phenolic hydroxyl moieties, which are in similar environments.

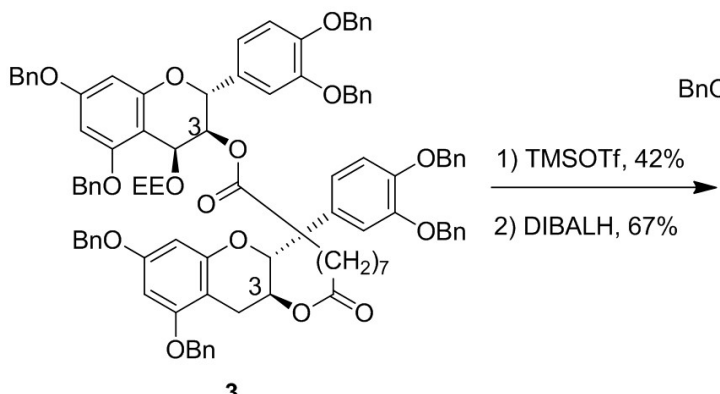

3

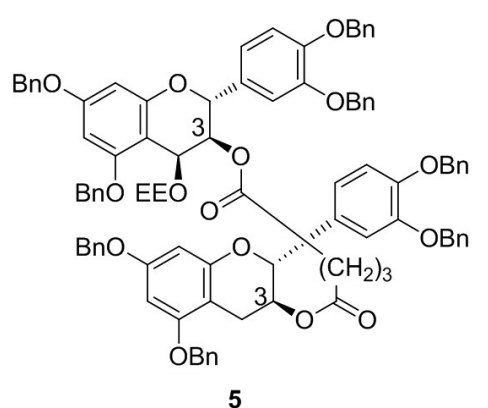

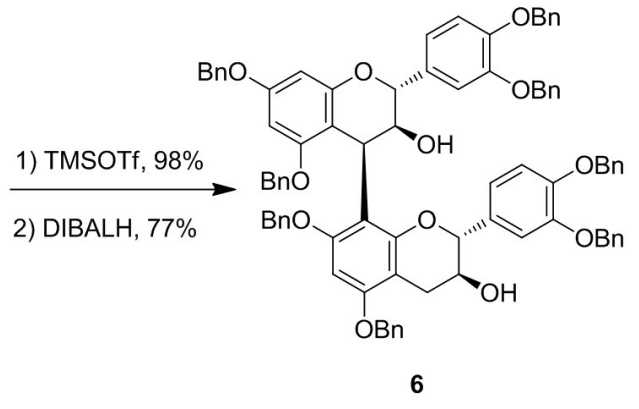

Scheme 1. Stereoselective synthesis of the basic skeleton of 3,4-trans and 3,4-cis-(+)-catechin dimers via an intramolecular coupling.

A thorough examination of the reactive properties of the protected flavan-3-ols revealed that the 5-O-TBDMS (TBS) group of the 5,7,3', $4^{\prime}$-tetra-O-TBDMS-(+)-catechin (7) could be regioselectively removed with TFA to give 8 in $90 \%$ yield [40] (Scheme 2). The structure of the 5-OH product (8) was confirmed by the HMBC (Heteronuclear Multiple Bond Correlation) between C5 and the hydroxyl proton of 8 [40].

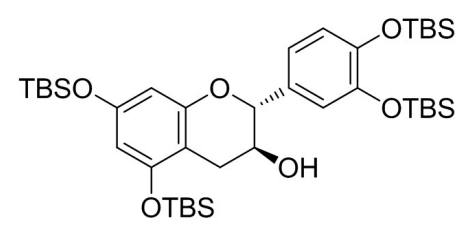

7

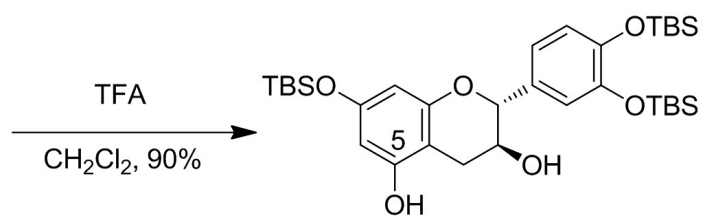

8

Scheme 2. Regioselective deprotection of the 5-O-t-butyldimethylsilyl (TBDMS) group.

Using the aforementioned regioselective deprotection method, the intramolecular synthesis of the 4-6-condensed procyanidin B6 (1) was achieved (Scheme 3). During this synthesis, the 5-O-TBDMS group of an acetylation derivative of 7 (9) was removed with TFA to give 5-hydroxyl nucleophile 10 in $76 \%$ yield. The 5-hydroxyl group of $\mathbf{1 0}$ was then esterified with azelaic acid using the $N, N^{\prime}$-dicyclohexylcarbodiimide (DCC) condensation method to afford the carboxylic acid derivative 11 in $80 \%$ yield. The 3-hydroxyl group of $\mathbf{1 2}$ (electrophile) [26] was then connected to 11 by esterification to obtain the diester compound 13. The subsequent $\mathrm{SnCl}_{4}$-catalyzed intramolecular condensation of $\mathbf{1 3}$ 
at $-20{ }^{\circ} \mathrm{C}$ proceeded smoothly to afford the $4-6$ condensed catechin dimer 14 in $72 \%$ yield. When the cyclization reaction was carried out with TMSOTf instead, another TBDMS group was also deprotected, resulting in a complicated mixture of many byproducts. Additionally, the use of dicarboxylic acids as linkers was unsuccessful, and the intramolecular cyclization proceeded only when azelaic acid was employed. Using linkers of other lengths led to self-condensation products of electrophile 12, which once again caused the formation of a complicated mixture of byproducts. Temperature optimization studies of the cyclization reaction showed that the yield was the highest when the reaction was carried out at $-20{ }^{\circ} \mathrm{C}$. A decrease in the yield was observed both at higher and lower temperatures. The subsequent removal of the diester linker in $\mathbf{1 4}$ via diisobutylaluminum hydrate (DIBAL) reduction gave triol 15 in $66 \%$ yield. Due to peak broadening in the NMR spectrum of 15, the tri-acetylated compound 16 was prepared in order to determine the dimeric structure and 3,4-trans stereochemistry of the synthesized product (Figure 2). Compound 15 was eventually converted to 3,4-trans-(+)-catechin-(4-6)-(+)-catechin dimer, procyanidin B6 (1), after TBDMS-group deprotection by tetrabutylammonium fluoride (TBAF) (85\% yield) and subsequent $\mathrm{Pd}(\mathrm{OH})_{2}$-catalyzed hydrogenolysis (65\% yield). The full spectroscopic data confirmed the structure of procyanidin B6 (1) [41].

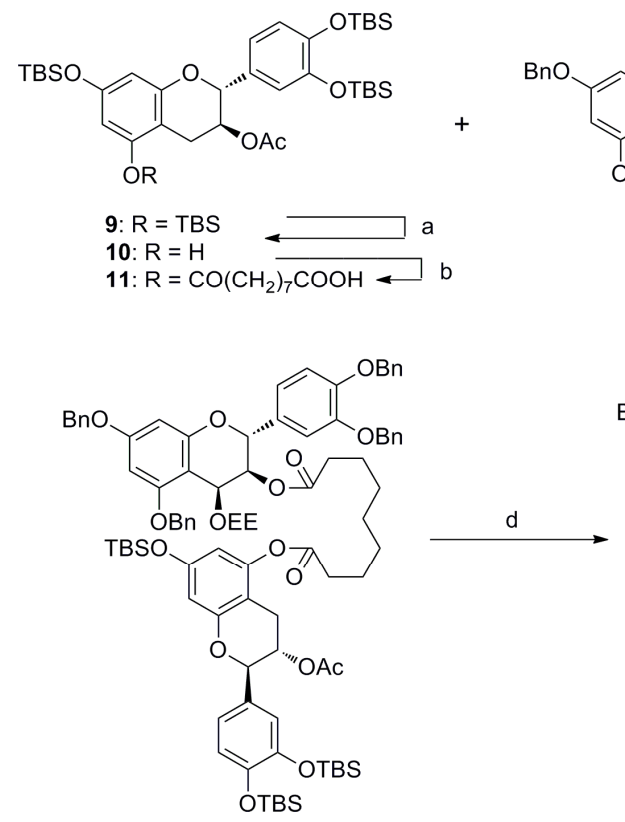

13

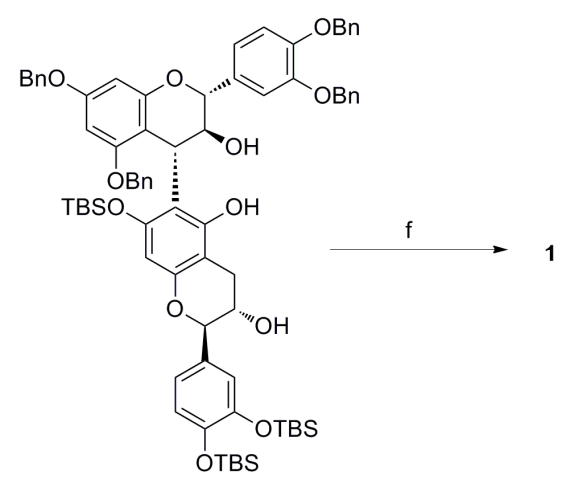

15

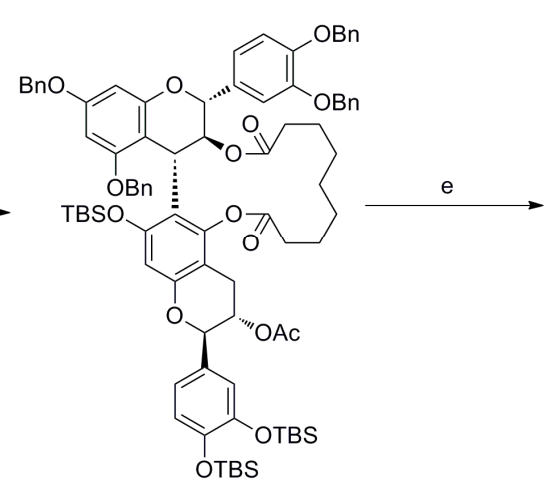

14

Scheme 3. Stereoselective synthesis of the procyanidin B6 (1). Reaction conditions: (a) TFA, $\mathrm{CH}_{2} \mathrm{Cl}_{2}$; $76 \%$ yield; (b) Azelaic acid, DCC, DMAP, $\mathrm{CH}_{2} \mathrm{Cl}_{2} ; 80 \%$ yield; (c) DCC, DMAP, $\mathrm{CH}_{2} \mathrm{Cl}_{2} ; 58 \%$ yield; (d) $\mathrm{SnCl}_{4}, \mathrm{CH}_{2} \mathrm{Cl}_{2},-20{ }^{\circ} \mathrm{C} ; 72 \%$ yield; (e) DIBALH, $\mathrm{CH}_{2} \mathrm{Cl}_{2} ; 66 \%$ yield; (f) (1) TBAF, $\mathrm{AcOH}$, THF, r.t.; $85 \%$ yield; (2) $\mathrm{Pd}(\mathrm{OH})_{2} / \mathrm{C}, \mathrm{H}_{2}, \mathrm{THF} / \mathrm{MeOH} / \mathrm{H}_{2} \mathrm{O}(20 / 1 / 1) ; 65 \%$ yield. 


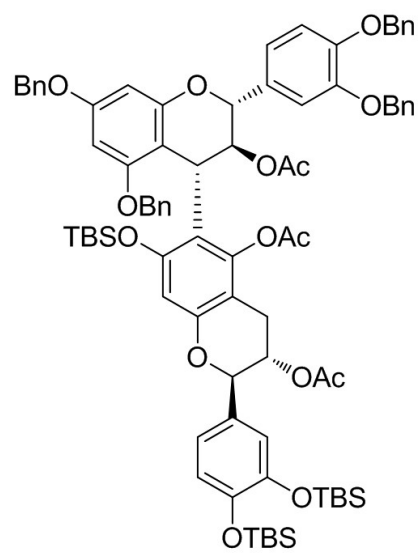

16

Figure 2. Acetylation derivative 16.

In addition, the coupling reaction of nucleophile $\mathbf{8}$ and electrophile $\mathbf{1 2}$ led to the formation of 4-6 and 4-8-condensed compounds (Scheme 4). After the intermolecular coupling products were acetylated, 16 and 17 were isolated and their structures were confirmed. Compound 16, which was synthesized via intramolecular coupling, was identified with the compound resulting from the intermolecular coupling. Thus, the intramolecularly cyclized product is a 4-6-connected dimer. Usually, when the 5-position is protected, a 4-6-condensed compound cannot be obtained. Therefore, it is possible that the electrophile was brought close to the 6-position through 5-TBDMS group deprotection. However, reactivity at the 6-position was extremely low as compared to that at the 8-position, and the ratio of the 4-6 and 4-8-condensed products was 1:9.

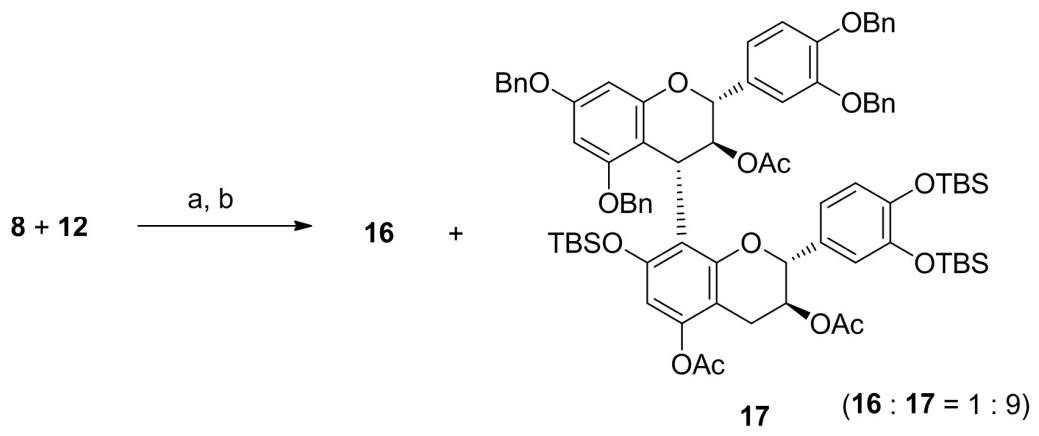

Scheme 4. Intermolecular coupling of 8 and 12. Reaction conditions: (a) TMSOTf, $\mathrm{CH}_{2} \mathrm{Cl}_{2},-40{ }^{\circ} \mathrm{C}$; $78 \%$ yield; (b) Acetic anhydride, DMAP, pyridine, quant.

\section{Materials and Methods}

All commercially available chemicals for the chemical syntheses were used without further purification. All reactions were performed under argon atmosphere and monitored with thin-layer chromatography (TLC) using $0.25 \mathrm{~mm}$ pre-coated silica gel plates 60F254 (Art 5715, Merck KGaA, Darmstadt, Germany). An ATAGO (Minato, Tokyo, Japan) AP-300 spectrometer was used to measure the optical rotation. ${ }^{1} \mathrm{H}$ - and ${ }^{13} \mathrm{C}-\mathrm{NMR}$ spectra were recorded on an Agilent Inova 500 Spectrometer (500/125 MHz) and DD2 NMR Spectrometer (400/100 MHz) (Agilent, Santa Clara, CA, USA). A JEOL JMS-AX500 mass spectrometer was used to acquire the fast atom bombardment (FAB) mass spectra. A microTOFfocus mass spectrometer (Bruker Daltonics, Billerica, MA, USA) was used to acquire the electrospray ionization (ESI) mass spectra. High-performance liquid chromatography (HPLC) purifications were carried out on an Ascentis ${ }^{\circledR}$ column (SUPELCO ${ }^{\circledR}$ analytical, Sigma Aldrich Co., St. Louis, MO, USA; $25 \mathrm{~cm} \times 21.5 \mathrm{~mm}, 5 \mu \mathrm{m}$ ) using the solvents (A) $0.05 \% \mathrm{HCOOH}$ in $\mathrm{CH}_{3} \mathrm{CN}$, 
and (B) $0.05 \% \mathrm{HCOOH}$ and $10 \% \mathrm{CH}_{3} \mathrm{CN}$ in $\mathrm{H}_{2} \mathrm{O}$. Elution was performed with a linear gradient $20-100 \% \mathrm{~B}$ in $25 \mathrm{~min}(4.0 \mathrm{~mL} / \mathrm{min}$ flow rate).

3-Acetoxy-3', 4',7-tri(t-butyldimethylsilyloxy)-(+)-catechin (10). To a solution of $9(0.27 \mathrm{~g}, 0.34 \mathrm{mmol})$ in $\mathrm{CH}_{2} \mathrm{Cl}_{2}(50 \mathrm{~mL})$ was added TFA $(0.031 \mathrm{~mL}, 0.41 \mathrm{mmol})$ slowly at $0{ }^{\circ} \mathrm{C}$. After stirring for $6 \mathrm{~h}$, the reaction mixture was quenched with sat. $\mathrm{NaHCO}_{3}$ solution in water. The aqueous solution was extracted with $\mathrm{CHCl}_{3}$, and the organic phase was washed with water and brine and then dried $\left(\mathrm{MgSO}_{4}\right)$. Filtration, concentration, and silica-gel column purification ( $n$-hexane/EtOAc, 10:1 to 3:1) afforded $0.18 \mathrm{~g}(0.26 \mathrm{mmol}, 76 \%)$ of $\mathbf{1 0}$ as an amorphous solid. $[\alpha]_{\mathrm{D}}^{24}+61.8\left(c 0.21, \mathrm{CHCl}_{3}\right) ;{ }^{1} \mathrm{H}-\mathrm{NMR}$ $\left(500 \mathrm{MHz}, \mathrm{CDCl}_{3}\right) 6.74(1 \mathrm{H}, \mathrm{br}), 6.71(2 \mathrm{H}, \mathrm{br}), 5.99(1 \mathrm{H}, \mathrm{d}, J=2.0 \mathrm{~Hz}), 5.89(1 \mathrm{H}, \mathrm{d}, J=2.0 \mathrm{~Hz}), 5.19(1 \mathrm{H}$, $\mathrm{ddd}, J=5.5,5.5,6.0 \mathrm{~Hz}), 4.92(1 \mathrm{H}, \mathrm{d}, J=5.5 \mathrm{~Hz}), 2.73(1 \mathrm{H}, \mathrm{dd}, J=5.5,7.0 \mathrm{~Hz}), 2.59(1 \mathrm{H}, \mathrm{dd}, J=6.0$, $17.0 \mathrm{~Hz}), 2.35(1 \mathrm{H}, \mathrm{br}), 1.88(3 \mathrm{H}, \mathrm{s}), 0.89-0.83(27 \mathrm{H}, \mathrm{m}), 0.11(9 \mathrm{H}, \mathrm{s}), 0.09(3 \mathrm{H}, \mathrm{s}), 0.05(3 \mathrm{H}, \mathrm{s}), 0.03(3 \mathrm{H}, \mathrm{s})$; ${ }^{13} \mathrm{C}-\mathrm{NMR}\left(125 \mathrm{MHz}, \mathrm{CDCl}_{3}\right)$ 170.5, 155.5, 155.0, 154.6, 147.0, 146.8, 131.0, 121.1, 119.4, 119.1, 100.6, $100.3,100.0,77.8,69.3,25.9(2), 25.7,23.1,21.1,-4.06,-4.11,-4.15,-4.24,-4.40,-4.43$; FABMS $(\mathrm{m} / \mathrm{z})$ : 676 (12), $675\left(\mathrm{M}^{+}+\mathrm{H}, 12\right), 674$ (6), 617 (13), 616 (23), 615 (45); 614 (23), 613 (14), 558 (10), 557 (20), 444 (10), 443 (29), 368 (26), 367 (81), 351 (66), 277 (28), 254 (18), 253 (86), 205 (16), 179 (100); HRFABMS: Calcd. for $\mathrm{C}_{35} \mathrm{H}_{59} \mathrm{O}_{7} \mathrm{Si}_{3}, 675.3569$; Found, 675.3563.

5-Azelaic acid ester (11). To a solution of $\mathbf{1 0}(0.47 \mathrm{~g}, 0.70 \mathrm{mmol})$ and azelaic acid $(0.39 \mathrm{~g}, 2.00 \mathrm{mmol})$ was added DCC $(0.17 \mathrm{~g}, 0.83 \mathrm{mmol})$ and cat. DMAP in $\mathrm{CH}_{2} \mathrm{Cl}_{2}(50 \mathrm{~mL})$ at $0{ }^{\circ} \mathrm{C}$. After stirring for $24 \mathrm{~h}$ at r.t., the reaction mixture was quenched with water. The aqueous solution was extracted with $\mathrm{CHCl}_{3}$, and the organic phase was washed with water and brine and then dried $\left(\mathrm{MgSO}_{4}\right)$. Filtration, concentration, and silica gel column purification $\left(\mathrm{CHCl}_{3} / \mathrm{EtOAc}, 200: 1\right.$ to 2:1 followed by n-hexane/EtOAc, 10:1 to 1:1) afforded $0.47 \mathrm{~g}(1.60 \mathrm{mmol}, 80 \%)$ of $\mathbf{1 1}$ as an amorphous solid. $[\alpha]_{\mathrm{D}}^{24}$ +196.2 (c 0.50, $\left.\mathrm{CHCl}_{3}\right) ;{ }^{1} \mathrm{H}-\mathrm{NMR}\left(400 \mathrm{MHz}, \mathrm{CDCl}_{3}\right) 10.40-10.10(1 \mathrm{H}, \mathrm{br}), 6.80(1 \mathrm{H}, \mathrm{d}, J=8.3 \mathrm{~Hz})$, $6.80(1 \mathrm{H}, 1.8 \mathrm{~Hz}), 6.77(1 \mathrm{H}, \mathrm{dd}, J=1.8,8.3 \mathrm{~Hz}), 6.36(1 \mathrm{H}, \mathrm{d}, J=2.4 \mathrm{~Hz}), 6.23(1 \mathrm{H}, \mathrm{d}, J=2.4 \mathrm{~Hz})$, $5.23(1 \mathrm{H}, \mathrm{ddd}, J=5.2,6.3,6.4 \mathrm{~Hz}), 2.75(1 \mathrm{H}, \mathrm{dd}, J=5.2,16.5 \mathrm{~Hz}), 2.56(1 \mathrm{H}, \mathrm{dd}, J=6.4,16.5 \mathrm{~Hz}), 2.51(2 \mathrm{H}$, $\mathrm{t}, J=7.7 \mathrm{~Hz}), 2.35(2 \mathrm{H}, \mathrm{t}, J=7.5 \mathrm{~Hz}), 1.95(3 \mathrm{H}, \mathrm{s}), 1.78-1.60(4 \mathrm{H}, \mathrm{m}), 1.43-1.30(6 \mathrm{H}, \mathrm{m}), 0.97(9 \mathrm{H}, \mathrm{s})$, $0.96(9 \mathrm{H}, \mathrm{s}), 0.94(9 \mathrm{H}, \mathrm{s}), 0.20(6 \mathrm{H}, \mathrm{s}), 0.185(3 \mathrm{H}, \mathrm{s}), 0.175(3 \mathrm{H}, \mathrm{s}), 0.13(3 \mathrm{H}, \mathrm{s}), 0.12(3 \mathrm{H}, \mathrm{s}) ;{ }^{13} \mathrm{C}-\mathrm{NMR}$ $\left(100 \mathrm{MHz}, \mathrm{CDCl}_{3}\right) 171.4,171.3,170.4,155.3,154.9,149.9,147.1,147.0,130.8,121.2,119.6,119.3,107.4$, $105.7,105.6,78.2,69.0,34.3,34.0,29.02,28.96,28.94,26.04,26.03,25.7,24.9,24.7,23.9,21.6,18.6,18.3$, -3.95,-3.98,-4.05,-4.10,-4.32,-4.35; ESIMS (m/z) 1153 (61), 1152 (68), 1151 (80), $1076(43)$, 1075 (79), 1074 (100), 869 (18), 868 (37), 687 ([M + Na] $]^{+}$, 56); ESIHRMS Calcd. for $\mathrm{C}_{44} \mathrm{H}_{72} \mathrm{O}_{10} \mathrm{Si}_{3} \mathrm{Na}$, 867.4331; Found, 867.4340.

Cyclization precursor 13. To a solution of $\mathbf{1 1}(0.34 \mathrm{~g}, 0.40 \mathrm{mmol})$ and $\mathbf{1 2}(0.77 \mathrm{~g}, 1.00 \mathrm{mmol})$ was added DCC $(99.0 \mathrm{mg}, 0.48 \mathrm{mmol})$ and catalytic amount of DMAP in $\mathrm{CH}_{2} \mathrm{Cl}_{2}(20 \mathrm{~mL})$ at $0{ }^{\circ} \mathrm{C}$. After stirring for $24 \mathrm{~h}$ at r.t., the reaction mixture was quenched with water. The aqueous solution was extracted with $\mathrm{CHCl}_{3}$, and the organic phase was washed with water and brine and then dried $\left(\mathrm{MgSO}_{4}\right)$. Filtration, concentration, and silica gel column purification (n-hexane/EtOAc, 10:1 to 3:1) afforded $0.37 \mathrm{~g}(0.24 \mathrm{mmol}, 58 \%)$ of $\mathbf{1 3}$ as an amorphous solid. $[\alpha]_{\mathrm{D}}^{24}+91.0\left(\mathrm{c} 0.61, \mathrm{CHCl}_{3}\right) ;{ }^{1} \mathrm{H}-\mathrm{NMR}(400 \mathrm{MHz}$, $\left.\mathrm{CDCl}_{3}\right) 747-7.28(20 \mathrm{H}, \mathrm{m}), 7.07(1 \mathrm{H}, \mathrm{d}, J=1.5 \mathrm{~Hz}), 6.98(1 \mathrm{H}, \mathrm{dd}, J=1.5,8.3 \mathrm{~Hz}), 6.92(1 \mathrm{H}, \mathrm{d}, J=8.3 \mathrm{~Hz})$, $6.80(1 \mathrm{H}, \mathrm{d}, J=1.5 \mathrm{~Hz}), 6.80(1 \mathrm{H}, \mathrm{dd}, J=1.5,8.3 \mathrm{~Hz}), 6.78(1 \mathrm{H}, \mathrm{d}, J=8.3 \mathrm{~Hz}), 6.36(1 \mathrm{H}, \mathrm{d}, J=2.4 \mathrm{~Hz})$, $6.24(1 \mathrm{H}, \mathrm{d}, J=2.0 \mathrm{~Hz}), 6.22(1 \mathrm{H}, \mathrm{d}, J=2.4 \mathrm{~Hz}), 6.13(1 \mathrm{H}, \mathrm{d}, J=2.0 \mathrm{~Hz}), 5.27(2 \mathrm{H}, \mathrm{s}), 5.26-5.21(1 \mathrm{H}, \mathrm{m})$, $5.14(2 \mathrm{H}, \mathrm{s}), 5.13(2 \mathrm{H}, \mathrm{s}), 5.16(1 \mathrm{H}, \mathrm{d}, J=11.6 \mathrm{~Hz}), 5.02(1 \mathrm{H}, \mathrm{d}, J=11.6 \mathrm{~Hz}), 5.00-4.87(3 \mathrm{H}, \mathrm{m}), 4.85(1 \mathrm{H}$, br s), 3.80-3.70 (2H, m), 3.49-3.37 (4H, m), $2.74(1 \mathrm{H}, \mathrm{dd}, J=5.2,16.4 \mathrm{~Hz}), 2.51(1 \mathrm{H}, \mathrm{dd}, J=6.8,16.4 \mathrm{~Hz})$, $2.43(2 \mathrm{H}, \mathrm{t}, J=7.6 \mathrm{~Hz}), 2.13-1.94(2 \mathrm{H}, \mathrm{m}), 1.92(3 \mathrm{H}, \mathrm{s}), 1.71-1.06(10 \mathrm{H}, \mathrm{m}), 0.974(9 \mathrm{H}, \mathrm{s}), 0.965(9 \mathrm{H}, \mathrm{s})$, $0.94(9 \mathrm{H}, \mathrm{s}), 0.20(6 \mathrm{H}, \mathrm{s}), 0.19(3 \mathrm{H}, \mathrm{s}), 0.18(3 \mathrm{H}, \mathrm{s}), 0.14(3 \mathrm{H}, \mathrm{s}), 0.12(3 \mathrm{H}, \mathrm{s}) ;{ }^{13} \mathrm{C}-\mathrm{NMR}(100 \mathrm{MHz}$, $\mathrm{CDCl}_{3}$ ) 172.5, 171.4, 170.2, 161.0, 158.6, 155.9, 155.3, 155.0, 149.9, 149.4, 149.1, 147.1, 147.0, 137.33, 137.30, 136.74, 136.66, 130.8, 130.7, 128.8, 128.7, 128.6, 128.24, 128.19, 128.95, 128.92, 128.88, 127.7, 127.6, 127.3, 121.6, 121.2, 119.6, 119.3, 114.8, 114.6, 107.4, 105.7, 105.6, 103.8, 94.4, 93.9, 78.3, 74.5, 72.5, 71.42, 71.36, $71.0,70.6,70.2,69.9,68.9,68.7,66.5,34.24,34.19,29.06,29.04,28.9,26.0$ (2), 25.7, 25.0, 24.7, 24.0, 21.1, 
18.6 (2), 18.3, 15.4, -3.94, -3.97, -4.04, -4.09, -4.31, -4.34; ESIMS (m/z) 1591 (29), 1590 (59), 1589 (89), $1588\left([\mathrm{M}+\mathrm{Na}]^{+}, 76\right) ; 472(30), 471$ (100); ESIHRMS Calcd. for $\mathrm{C}_{91} \mathrm{H}_{116} \mathrm{O}_{17} \mathrm{Si}_{3} \mathrm{Na}, 1587.7418$; Found, 1587.7413.

Cyclic compound 14. To a solution of $13(0.17 \mathrm{~g}, 0.10 \mathrm{mmol})$ in $\mathrm{CH}_{2} \mathrm{Cl}_{2}(60 \mathrm{~mL})$ at $-10{ }^{\circ} \mathrm{C}$ was added $\mathrm{SnCl}_{4}\left(0.48 \mathrm{~mL}, 0.12 \mathrm{mmol}, 0.25 \mathrm{M}\right.$ solution in $\left.\mathrm{CH}_{2} \mathrm{Cl}_{2}\right)$. After stirring for $5 \mathrm{~min}$, the reaction mixture was quenched with sat. $\mathrm{NaHCO}_{3}$ in water. The aqueous solution was extracted with $\mathrm{CHCl}_{3}$, and the organic phase was washed with water and brine and then dried $\left(\mathrm{MgSO}_{4}\right)$. Filtration, concentration, and silica-gel PTLC purification ( $n$-hexane/EtOAc, 5:1) afforded $0.11 \mathrm{~g}(0.072 \mathrm{mmol}, 72 \%)$ of 14 as an amorphous solid. $[\alpha]_{\mathrm{D}}^{24}+130\left(c\right.$ 0.20, $\left.\mathrm{CHCl}_{3}\right) ;{ }^{1} \mathrm{H}-\mathrm{NMR}\left(500 \mathrm{MHz}, \mathrm{CDCl}_{3}\right) 7.50-7.28(21 \mathrm{H}, \mathrm{m}), 7.17(1 \mathrm{H}$, $\mathrm{d}, J=1.5 \mathrm{~Hz}), 6.96(1 \mathrm{H}, \mathrm{dd}, J=1.5,8.5 \mathrm{~Hz}), 6.90(1 \mathrm{H}, \mathrm{d}, J=8.5 \mathrm{~Hz}), 6.83(1 \mathrm{H}, \mathrm{d}, J=2.2 \mathrm{~Hz}), 6.76(1 \mathrm{H}, \mathrm{dd}$, $J=1.5,8.5 \mathrm{~Hz}), 6.31(1 \mathrm{H}, \mathrm{s}), 6.19(1 \mathrm{H}, \mathrm{d}, J=2.2 \mathrm{~Hz}), 6.09(1 \mathrm{H}, \mathrm{br} \mathrm{s}), 5.95(1 \mathrm{H}, \mathrm{t}, J=9.5 \mathrm{~Hz}), 5.20-4.90$ $(9 \mathrm{H}, \mathrm{m}), 4.74(1 \mathrm{H}, \mathrm{d}, J=9.5 \mathrm{~Hz}), 4.58(1 \mathrm{H}, \mathrm{d}, J=9.5 \mathrm{~Hz}), 2.70-2.60(1 \mathrm{H}, \mathrm{m}), 2.51(1 \mathrm{H}, \mathrm{dd}, J=8.5$, $15.5 \mathrm{~Hz}), 2.42-1.27(14 \mathrm{H}, \mathrm{m}), 1.57(3 \mathrm{H}, \mathrm{s}), 1.00(9 \mathrm{H}, \mathrm{s}), 0.98(9 \mathrm{H}, \mathrm{s}), 0.83(9 \mathrm{H}, \mathrm{s}), 0.29(6 \mathrm{H}, \mathrm{s}), 0.21(12 \mathrm{H}$, s) ${ }^{13} \mathrm{C}-\mathrm{NMR}\left(125 \mathrm{MHz} \mathrm{CDCl}_{3}\right)$ 171.8, 156.7, 158.5, 153.3, 152.7, 149.2, 149.1, 149.0, 146.9, 146.7, 137.3, 137.2, 137.0, 130.1-127.3 (C24), 121.0, 116.3, 114.6, 107.4, 103.9, 95.3, 94.8, 81.5, 78.0, 71.2, 71.23, 71.17, 70.1, 69.2, 37.7, 34.0, 32.0, 29.7, 26.9, 26.3, 26.2, 26.0, 25.9, 25.6, 21.0, 18.8, 18.4, 0.0, -3.4, -3.9, -4.10, -4.14; ESIMS (m/z) 1500 (30), 1499 (45), 1498 ([M + Na $\left.]^{+}, 41\right) ; 741(25), 740$ (55), 713 (44), 712 (100), 685 (20), 684 (49); ESIHRMS Calcd for $\mathrm{C}_{87} \mathrm{H}_{106} \mathrm{O}_{15} \mathrm{Si}_{3} \mathrm{Na}$, 1497.6737; Found, 1497.6732.

[4,6]-2,3-Trans-3,4-trans-2' ,3',5,7-tetra-O-benzyl-2"' ,3'"',7"-tri(t-butyldimethylsilyloxy)-(+)-catechin-(+)catechin (15). To a solution of $14(5.0 \mathrm{mg}, 0.0030 \mathrm{mmol})$ in $\mathrm{CH}_{2} \mathrm{Cl}_{2}(10 \mathrm{~mL})$ was reduced with DIBALH $\left(0.030 \mathrm{~mL}, 0.030 \mathrm{mmol}, 1.0 \mathrm{~mol}\right.$ solution in $n$-hexane) at $-78^{\circ} \mathrm{C}$. After stirring for $5 \mathrm{~min}$, the reaction mixture was quenched with sat. $\mathrm{NH}_{4} \mathrm{Cl}$ in water. The aqueous solution was extracted with $\mathrm{CHCl}_{3}$, and the organic phase was washed with water and brine and then dried $\left(\mathrm{MgSO}_{4}\right)$. Filtration, concentration, and silica-gel PTLC purification ( $n$-hexane/EtOAc, 2:1) afforded $3.0 \mathrm{mg}(0.20 \mathrm{mmol}$, $66 \%)$ of 15 as an amorphous solid. $[\alpha]_{\mathrm{D}}^{24}+80.0\left(c 0.05, \mathrm{CHCl}_{3}\right)$; The $\mathrm{NMR}$ signals were not identified because of peak broadening. FABMS $(m / z)$ : $1282\left(3.6, \mathrm{M}^{+}+\mathrm{H}\right), 1281(2.8), 1263$ (1.2), 933 (6.7), 932 (9.0), 931 (12), 844 (5.9), 843 (9.1), 842 (13), 723 (15), 722 (24), 650 (19), 649 (39), 393 (37), 352 (31), 351 (100); FABHRMS: $m / z\left(\mathrm{M}^{+}+\mathrm{H}\right)$ Calcd. for $\mathrm{C}_{76} \mathrm{H}_{93} \mathrm{O}_{12} \mathrm{Si}_{3}, 1281.5975$; Found, 1281.6067 .

[4,6]-2,3-Trans-3,4-trans-(+)-catechin-(+)-catechin (procyanidin B6) (1). A solution of 15 (32.0 mg, $0.025 \mathrm{mmol})$ in THF $(20 \mathrm{~mL})$ was added dropwise to TBAF $(0.87 \mathrm{~mL} ; 0.87 \mathrm{mmol} ; 1 \mathrm{M}$ solution in THF $)$ in the presence of $\mathrm{AcOH}(0.0050 \mathrm{~mL} ; 0.87 \mathrm{mmol})$ at $0{ }^{\circ} \mathrm{C}$. Concentration and PTLC purification (n-hexane/EtOAc; $2: 1)$ afforded $19.0 \mathrm{mg}$ of TBS deprotected product $(0.021 \mathrm{mmol}, 85 \%)$ as an amorphous solid. Then $15 \mathrm{mg}$ of the above product was dissolved in THF/MeOH/ $\mathrm{H}_{2} \mathrm{O}(20: 1: 1 ; 5.5 \mathrm{~mL})$ and hydrogenated over $20 \% \mathrm{Pd}(\mathrm{OH})_{2} / \mathrm{C}(1 \mathrm{mg})$ for $12 \mathrm{~h}$ at r.t. Filtration and concentration afforded a pale brown solid; which was purified using HPLC purification to give $6.0 \mathrm{mg}$ of 1 (0.010 mmol, $65 \%$ ) as a pale brown powder. ${ }^{1} \mathrm{H}-\mathrm{NMR}\left(400 \mathrm{MHz}, \mathrm{CDCl}_{3}, 0.75: 0.25\right.$ mixture of rotational isomers) major: $6.78(0.75 \mathrm{H}, \mathrm{d}, J=2.0 \mathrm{~Hz}), 6.72(0.75 \mathrm{H}, \mathrm{d}, J=8.2 \mathrm{~Hz}), 6.71(0.75 \mathrm{H}, \mathrm{d}, J=8.2 \mathrm{~Hz}), 6.63(0.75 \mathrm{H}, \mathrm{d}$, $J=2.0 \mathrm{~Hz}), 6.51(0.75 \mathrm{H}, \mathrm{dd}, J=2.0,8.2 \mathrm{~Hz}), 6.29(0.75 \mathrm{H}, \mathrm{dd}, J=2.0,8.2 \mathrm{~Hz}), 6.11(0.75 \mathrm{H}, \mathrm{s}), 5.93(0.75 \mathrm{H}$, $J=2.4 \mathrm{~Hz}), 5.83(0.75 \mathrm{H}, \mathrm{d}, J=2.4 \mathrm{~Hz}), 4.58(0.75 \mathrm{H}, \mathrm{d}, J=7.5 \mathrm{~Hz}), 4.45(0.75 \mathrm{H}, \mathrm{d}, J=7.8 \mathrm{~Hz}), 4.39(0.75 \mathrm{H}$, $\mathrm{dd}, J=7.8,9.5 \mathrm{~Hz}), 4.29(0.75 \mathrm{H}, \mathrm{d}, J=9.5 \mathrm{~Hz}), 3.86-3.81(0.75 \mathrm{H}, \mathrm{m}), 2.80(0.75 \mathrm{H}, \mathrm{dd}, J=5.7,16.2 \mathrm{~Hz})$, $2.52(0.75 \mathrm{H}, \mathrm{dd}, J=8.1,16.2 \mathrm{~Hz})$; monor: $700-6.73(1.5 \mathrm{H}, \mathrm{m}), 5.98(0.25 \mathrm{H}, \mathrm{s}), 5.88(0.25 \mathrm{H}, \mathrm{d}, J=2.4 \mathrm{~Hz})$, $5.85(0.25 \mathrm{H}, \mathrm{d}, J=2.4 \mathrm{~Hz}), 4.98-4.52(0.25 \mathrm{H}, \mathrm{m}), 4.18-4.08(0.25 \mathrm{H}, \mathrm{m}), 2.86(0.25 \mathrm{H}, \mathrm{dd}, J=5.7,16.4 \mathrm{~Hz})$, $2.62(0.25 \mathrm{H}, \mathrm{dd}, J=8.0,16.4 \mathrm{~Hz}) ;{ }^{13} \mathrm{C}-\mathrm{NMR}\left(100 \mathrm{MHz}, \mathrm{CDCl}_{3}\right)$ major: 158.2, 157.9, 157.2, 156.3, 155.3, 154.9, 146.7, 146.6, 146.5, 143.9, 132.54, 132.53, 129.8, 129.1, 126.4, 120.3, 116.4, 115.6, 110.0, 101.6, 101.1, $96.6,96.4,95.8,83.2,82.9,69.2,69.1,29.3,25.1$ (minor isomer was not identified).

[4,6]-2,3-Trans-3,4-trans-3,3"', ,5"'-triacetoxy-2',3',5,7-tetra-O-benzyl-2"' , $3^{\prime \prime \prime}, 7^{\prime \prime}$-tri(t-butyldimethylsilyl-oxy) $-(+)$-catechin-(+)-catechin (16). To a solution of $15(3.0 \mathrm{mg}, 0.0030 \mathrm{mmol})$ in $\mathrm{CH}_{2} \mathrm{Cl}_{2}$ was acetylated with excess $\mathrm{Et}_{3} \mathrm{~N}(8.3 \mu \mathrm{L}, 0.06 \mathrm{mmol})$ and acetic anhydride $(4.2 \mu \mathrm{L}, 0.045 \mathrm{mmol})$ in the presence of 
catalytic amount of DMAP at $0{ }^{\circ} \mathrm{C}$. After stirring for $24 \mathrm{~h}$, the reaction mixture was quenched with water. The aqueous solution was extracted with $\mathrm{CHCl}_{3}$, and the organic phase was washed with water and brine and then dried $\left(\mathrm{MgSO}_{4}\right)$. Filtration, concentration, and silica-gel PTLC purification (n-hexane/EtOAc, 3:1) afforded $2.0 \mathrm{mg}(0.0014 \mathrm{mmol}, 60 \%)$ of $\mathbf{1 6}$ as an amorphous solid. $[\alpha]_{\mathrm{D}}^{24}+24.9$ (c 0.40, $\left.\mathrm{CHCl}_{3}\right) ;{ }^{1} \mathrm{H}-\mathrm{NMR}\left(500 \mathrm{MHz}, \mathrm{CDCl}_{3}\right) 7.48-6.82(23 \mathrm{H}, \mathrm{m}), 7.99(1 \mathrm{H}, \mathrm{dd}, J=2.2,8.5 \mathrm{~Hz}), 6.91(1 \mathrm{H}$, d, $J=8.5 \mathrm{~Hz}), 6.88(1 \mathrm{H}, \mathrm{d}, J=2.2 \mathrm{~Hz}), 5.65(1 \mathrm{H}, \mathrm{t}, J=9.5 \mathrm{~Hz}), 6.20(1 \mathrm{H}, \mathrm{d}, J=2.5 \mathrm{~Hz}), 6.16(1 \mathrm{H}, \mathrm{d}$, $J=2.5 \mathrm{~Hz}), 6.03(1 \mathrm{H}, \mathrm{s}), 5.21-5.12(5 \mathrm{H}, \mathrm{m}), 5.99(1 \mathrm{H}, \mathrm{d}, J=12.5 \mathrm{~Hz}), 4.95(1 \mathrm{H}, \mathrm{d}, J=12.5 \mathrm{~Hz}), 4.87(1 \mathrm{H}$, $\mathrm{d}, J=8.0 \mathrm{~Hz}), 4.82(1 \mathrm{H}, \mathrm{d}, J=9.5 \mathrm{~Hz}), 4.69(1 \mathrm{H}, \mathrm{d}, J=9.5 \mathrm{~Hz}), 4.64(2 \mathrm{H}, \mathrm{s}), 2.87(1 \mathrm{H}, \mathrm{dd}, J=6.0$, $16.0 \mathrm{~Hz}), 2.49(1 \mathrm{H}, \mathrm{dd}, J=8.0,16.0 \mathrm{~Hz}), 1.83(3 \mathrm{H}, \mathrm{s}), 1.70(3 \mathrm{H}, \mathrm{s}), 1.54(3 \mathrm{H}, \mathrm{s}), 0.98(18 \mathrm{H}, \mathrm{s}), 0.91(9 \mathrm{H}, \mathrm{s})$, $0.00(18 \mathrm{H}, \mathrm{s}) ;{ }^{13} \mathrm{C}-\mathrm{NMR}\left(125 \mathrm{MHz}, \mathrm{CDCl}_{3}\right)$ 169.7, 168.7, 167.4, 158.7, 158.6, 156.3, 153.5, 152.9, 149.2, 148.7, 147.9, 147.2, 146.8, 137.3, 137.2, 136.9, 136.6, 130.7, 130.1, 128.5-127.3 (C17), 121.2, 121.1, 120.2, 106.7, 103.5, 95.2, 95.0, 80.4, 79.1, 73.1, 71.5, 71.1, 70.8, 69.9, 69.2, 35.8, 29.7, 26.0, 25.9, 25.6, 22.7, 20.9, 20.5, 19.8, 18.5, 18.1, 14.2, -4.0, -4.1 (2); FABMS (m/z): $1408\left(\mathrm{M}^{+}+\mathrm{H}, 25\right), 1308(28), 1305$ (77), 1258 (30), 1257 (43), 1256 (44), 1216 (28), 1215 (52), 1214 (78), 1156 (27), 1155 (31), 1154 (37), 977 (21), 976 (23), 975 (33), 885 (23), 884 (21), 883 (31), 793 (29), 792 (31), 791 (48), 695 (40), 693 (29), 692 (52), 691 (100), 689 (35); HRFABMS: Calcd. for $\mathrm{C}_{82} \mathrm{H}_{99} \mathrm{O}_{15} \mathrm{Si}_{3}, 1407.6392$; Found, 1407.6284.

[4,8]-2,3-Trans-3,4-trans-2",3"-trans-3,5,3"-tri-O-acetyl-5,7,3',4'-tetra-O-benzyl-7", 3'", ,4'"'-tri-O-TBDMS-(+) -catechin-(+)-catechin (17). Acetylation product of intermolecular condensation product of 8 and 12: Data for 17: $[\alpha]_{\mathrm{D}}^{23}+1.3\left(c 0.35, \mathrm{CHCl}_{3}\right) ;{ }^{1} \mathrm{H}-\mathrm{NMR}\left(500 \mathrm{MHz}, \mathrm{CDCl}_{3}, 0.62\right.$ : 0.38 mixture of rotational isomers) major: 7.49-6.76 $(15.5 \mathrm{H}, \mathrm{m}), 6.56(0.62 \mathrm{H}, \mathrm{dd}, J=2.0,8.0 \mathrm{~Hz}), 6.29(0.62 \mathrm{H}, \mathrm{s}), 6.25(0.62 \mathrm{H}, \mathrm{d}$, $J=2.0 \mathrm{~Hz}), 6.21(0.62 \mathrm{H}, \mathrm{d}, J=2.0 \mathrm{~Hz}), 5.93(0.62 \mathrm{H}, \mathrm{t}, J=10.0 \mathrm{~Hz}), 5.19-4.78(4.34 \mathrm{H}, \mathrm{m}), 4.75(0.62 \mathrm{H}$, $\mathrm{d}, J=10.0 \mathrm{~Hz}), 4.67(0.62 \mathrm{H}, \mathrm{d}, J=11.0 \mathrm{~Hz}), 4.55(0.62 \mathrm{H}, \mathrm{d}, J=11.0 \mathrm{~Hz}), 3.41(0.62 \mathrm{H}, \mathrm{d}, J=8.5 \mathrm{~Hz})$, $2.97(0.62 \mathrm{H}, \mathrm{dd}, J=6.5,16.0 \mathrm{~Hz}), 2.36-2.24(0.62 \mathrm{H}, \mathrm{m}), 2.33(1.86 \mathrm{H}, \mathrm{s}), 1.80(1.86 \mathrm{H}, \mathrm{s}), 1.63(1.86 \mathrm{H} . \mathrm{s})$, $1.01(11.16 \mathrm{H}, \mathrm{s}), 0.86(5.58 \mathrm{H}, \mathrm{s}), 0.279-0.038(11.16 \mathrm{H}, \mathrm{m})$; minor: $7.49-6.76(9.12 \mathrm{H}, \mathrm{m}), 6.63(0.38 \mathrm{H}, \mathrm{d}$, $J=2.0 \mathrm{~Hz}), 6.18(0.38 \mathrm{H}, \mathrm{s}), 6.11(0.38 \mathrm{H}, \mathrm{d}, J=2.0 \mathrm{~Hz}), 6.00(0.38 \mathrm{H}, \mathrm{d}, J=2.0 \mathrm{~Hz}), 5.95(0.38 \mathrm{H}, \mathrm{dd}, J=2.0$, $8.0 \mathrm{~Hz}), 5.81(0.38 \mathrm{H}, \mathrm{t}, J=9.5 \mathrm{~Hz}), 5.19-4.78(3.8 \mathrm{H}, \mathrm{m}), 4.62(0.38 \mathrm{H}, \mathrm{d}, J=9.5 \mathrm{~Hz}), 2.77(0.38 \mathrm{H}, \mathrm{dd}, J=5.0$, $13.5 \mathrm{~Hz}), 2.61(0.38 \mathrm{H}, \mathrm{dd}, J=4.5,13.5 \mathrm{~Hz}), 2.28(1.14 \mathrm{H}, \mathrm{s}), 1.96(1.14 \mathrm{H}, \mathrm{s}), 1.58(1.14 \mathrm{H}, \mathrm{s}), 1.03(3.42 \mathrm{H}, \mathrm{s})$, $0.97(3.42 \mathrm{H}, \mathrm{s}), 0.93(3.42 \mathrm{H}, \mathrm{S}), 0.28-0.038(6.84 \mathrm{H}, \mathrm{m}) ;{ }^{13} \mathrm{C}-\mathrm{NMR}\left(125 \mathrm{MHz}, \mathrm{CDCl}_{3}, 0.62: 0.38\right.$ mixture of rotational isomers) major: 169.8, 169.2, 168.8, 158.7, 158.3, 156.5, 154.5, 153.0, 149.34, 149.27, 147.5, 146.8, 146.3, 137.6, 137.5, 137.3, 131.2, 129.2, 128.8-127.3 (C24), 121.8, 120.8, 120.6, 119.9, 116.8, 115.0, 114.4, 107.7, 105.6, 105.1, 95.5, 94.7, 80.7, 78.0, 71.5 (C2), 70.3, 70.1, 69.8, 36.0, 27.0, 26.4, 26.24, 26.23, 21.3, 21.2, 21.0, 19.0, 18.8, 18.6, 1.0-0 (C3); minor: 170.8, 169.1, 168.6, 158.5, 157.7, 157.1, 153.4, 153.1, 149.3, 148.7, 147.7, 147.2, 146.7, 137.6, 137.4, 136.8, 130.83, 130.76, 128.8-127.3 (Cx24), 121.24, 121.18, 119.7, 119.3, 117.5, 115.2, 115.0, 107.8, 105.7, 104.4, 95.0, 94.9, 80.3, 77.8, 71.8, 71.4, 70.7, 70.1, 69.5, 36.0, 26.23, 26.21, 26.1, 24.4, 21.2, 20.9, 20.8, 18.72, 18.67, 18.5, 1.0-0 (C3); FABMS (m/z): $1408(26), 1407\left(\mathrm{M}^{+}+\mathrm{H}, 32\right)$, 1348 (43), 1347 (64), 1346 (61), 1345 (26), 1257 (66), 1256 (100), 1255 (94), 1196 (34), 1195 (38); FABHRMS: Calcd. for $\mathrm{C}_{82} \mathrm{H}_{99} \mathrm{O}_{15} \mathrm{Si}_{3}, 1407.6292$; Found, 1407.6226.

\section{Conclusions}

In conclusion, we developed a new and simple synthetic method to obtain the 4-6-connected (+)-catechin dimer, procyanidin B6 (1), without capping the C-8 position. By regioselectively deprotecting the 5-position of TBDMS-protected flavan-3-ols, it was possible to connect the electrophile to the 5-position of the nucleophile via a diester linker. When azelaic acid was used as a diester linker, the intramolecular coupling reaction proceeded smoothly, and the desired 4-6 condensed (+)-catechin dimer, procyanidin B6 (1), was selectively obtained. Through this synthetic method, stereoselectivity could be obtained merely by esterification and condensation. 
Acknowledgments: This work was supported by BRAIN: the Program for Promotion of Basic and Applied Research for Innovations in Bio-oriented Industry (to A. Saito), the Technology Research Promotion Program for Agriculture, Forestry, Fisheries, Food industry (to A. Saito), and JSPS KAKENHI Grant Number 23780123 (Grant-in-Aid for Young Scientist (B) to A. Saito) and Grant Number 24590014 (Grants-in-Aid for Scientific Research (C) to N. Nakajima).

Author Contributions: Y.H., T.O., and K.M. synthesized all compounds; T.K., M.H., N.N., and A.S. supervised the study; Y.H. and A.S. wrote the manuscript.

Conflicts of Interest: The authors declare no conflict of interest.

$\begin{array}{ll}\text { Abbreviations } & \\ \text { AcOH } & \text { acetic acid } \\ \text { Bn } & \text { benzyl } \\ \text { DCC } & N_{\text {, }} \text {-dicyclohexylcarbodiimide } \\ \text { DIBALH } & \text { diisobutylaluminum hydrate } \\ \text { DMAP } & \text { 4-dimethylaminopyridine } \\ \text { DMSO } & \text { dimethyl sulfoxide } \\ \text { EE } & \text { ethoxyethyl } \\ \text { ESI } & \text { electrospray ionization } \\ \text { Et }{ }_{3} N & \text { trimethylamine } \\ \text { EtOAc } & \text { ethyl acetate } \\ \text { FAB } & \text { fast atom bombardment } \\ \text { HPLC } & \text { high-performance liquid chromatography } \\ \text { MeOH } & \text { methanol } \\ \text { SAR } & \text { structure-activity-relationship studies } \\ \text { TBAF } & \text { tetrabutylammonium fluoride } \\ \text { TBDMS and TBS } & t \text {-butyldimethylsilyl } \\ \text { TFA } & \text { trifluoroacetic acid } \\ \text { THF } & \text { tetrahydrofuran } \\ \text { TLC } & \text { thin-layer chromatography } \\ \text { TMSOTf } & \text { trimethylsilyl triflate }\end{array}$

\section{References}

1. Harborne, J.B. The Flavonoids: Advances in Research from 1986; Chapman and Hall: London, UK, 1993.

2. Harbone, J.B.; Baxter, H. The Handbook of Natural Flavonoids; John Wiley \& Sons: New York, NY, USA, 1999.

3. Oracz, J.; Żyżelewicz, D.; Nebesny, E. The content of polyphenolic compounds in cocoa beans (Theobroma cacao L.), depending on variety, growing region and processing operations: A review. Crit. Rev. Food Sci. Nutr. 2015, 55, 1176-1192. [CrossRef] [PubMed]

4. Żyżelewicz, D.; Zakłos-Szyda, M.; Juśkiewicz, J.; Bojczuk, M.; Oracz, J.; Budryn, G.; Miśkiewicz, K.; Krysiak, W.; Zduńczyk, Z.; Jurgoński, A. Cocoa bean (Theobroma cacao L.) phenolic extracts as PTP1B inhibitors, hepatic HepG2 and pancreatic $\beta$-TC3 cell cytoprotective agents and their influence on oxidative stress in rats. Food Res. Int. 2016, 89, 946-957. [CrossRef]

5. Cushnie, T.P.T.; Lamb, A.J. Antimicrobial activity of flavonoids. Int. J. Antimicrob. Agents 2005, 26, 343-356. [CrossRef] [PubMed]

6. Dhanasekaran, D.; Thajuddin, N.; Panneerselvam, A. Fungicides for Plant and Animal Diseases; InTech: Rijeka, Croatia, 2012.

7. Tsukuda, S.; Watashi, K.; Hojima, T.; Isogawa, M.; Iwamoto, M.; Omagari, K.; Suzuki, R.; Aizaki, H.; Kojima, S.; Sugiyama, M.; et al. A New class of hepatitis B and D virus entry inhibitors, proanthocyanidin and its analogs, that directly act on the viral large surface proteins. Hepatology 2017, 65, 1104-1116. [CrossRef] [PubMed]

8. Mena, P.; Domínguez-Perles, R.; Gironés-Vilaplana, A.; Baenas, N.; García-Viguera, C.; Villaño, D. Flavan-3-ols, anthocyanins, and inflammation. IUBMB Life 2014, 66, 745-758. [CrossRef] [PubMed]

9. Du, G.J.; Zhang, Z. Epigallocatechin gallate (EGCG) is the most effective cancer chemopreventive polyphenol in green tea. Nutrients 2012, 4, 1679-1691. [CrossRef] [PubMed] 
10. Wang, C.Z.; Mehendale, S.; Calway, T.; Yuan, C.S. Botanical flavonoids on coronary heart disease. Am. J. Chin. Med. 2011, 39, 661-671. [CrossRef] [PubMed]

11. Tanaka, T.; Matsuo, Y.; Kouno, I. Chemistry of secondary polyphenols produced during processing of tea and selected foods. Int. J. Mol. Sci. 2009, 11, 14-40. [CrossRef] [PubMed]

12. Johnson, M.C.; Thomas, A.L.; Greenlief, C.M. Impact of frozen storage on the anthocyanin and polyphenol content of American elderberry fruit juice. J. Agric. Food Chem. 2015, 63, 5653-5659. [CrossRef] [PubMed]

13. Delcour, J.A.; Tuytens, G.M. Structure elucidation of three demeric proanthocyanidins isolated from a commercial Belgan pilsner beer. J. Inst. Brew. 1984, 90, 153-161. [CrossRef]

14. Tückmantel, W.; Kozikowski, A.P.; Romanczyk, L.J. Studies in polyphenol chemistry and bioactivity. 1. Preparation of building blocks from (+)-catechin. Procyanidin formation. Synthesis of the cancer cell growth inhibitor, 3-O-galloyl-(2R,3R)-epicatechin-4 $\beta, 8-[3-O-g a l l o y l-(2 R, 3 R)$-epicatechin]. J. Am. Chem. Soc. 1999, 121, 12073-12081. [CrossRef]

15. Kozikowski, A.P.; Tückmantel, W.; George, C. Studies in polyphenol chemistry and bioactivity. 2. Establishment of interflavan linkage regio- and stereochemistry by oxidative degradation of an $O$-alkylated derivative of procyanidin $\mathrm{B}_{2}$ to (R)-(-)-2,4-diphenylbutyric acid. J. Org. Chem. 2000, 65, 5371-5381. [CrossRef] [PubMed]

16. Kozikowski, A.P.; Tückmantel, W.; Böttcher, G.; Romanczyk, L.J., Jr. Studies in polyphenol chemistry and bioactivity. 4. Synthesis of trimeric, tetrameric, pentameric, and higher oligomeric epicatechin-derived procyanidins having all-4 $\beta, 8$-interflavan connectivity and their inhibition of cancer cell growth through cell cycle arrest. J. Org. Chem. 2003, 68, 1641-1658. [PubMed]

17. Ohmori, K.; Ushimaru, N.; Suzuki, K. Oligomeric catechins: An enabling synthetic strategy by orthogonal activation and C(8) protection. Proc. Natl. Acad. Sci. USA 2004, 101, 12002-12007. [CrossRef] [PubMed]

18. Tarascou, I.; Barathieu, K.; Andre, Y.; Pianet, I.; Dufourc, E.; Fouquet, E. An improved synthesis of procyanidin dimers: Regio- and stereocontrol of the interflavan bond. Eur. J. Org. Chem. 2006, 23, 5367-5377. [CrossRef]

19. Sharma, P.K.; Kolchinski, A.; Shea, H.A.; Nair, J.J.; Gou, Y.; Romanczyk, L.J.; Schmitz, H.H. Scale-up syntheses of two naturally occurring procyanidins: (-)-epicatechin- $(4 \beta, 8)-(+)$-catechin and (-)-epicatechin-3-O-galloyl-(4ß,8)-(-)-epicatechin-3-O-gallate. Org. Process Res. Dev. 2007, 11, 422-430. [CrossRef]

20. Mohri, Y.; Sagehashi, M.; Yamada, T.; Hattori, Y.; Morimura, K.; Kamo, T.; Hirota, M.; Makabe, H. An efficient synthesis of procyanidins. Rare earth metal Lewis acid catalyzed equimolar condensation of catechin and epicatechin. Tetrahedron Lett. 2007, 48, 5891-5894. [CrossRef]

21. Oyama, K.; Kuwano, M.; Ito, M.; Yoshida, K.; Kondo, T. Synthesis of procyanidins by stepwiseand self-condensation using 3,4-cis-4-acetoxy-3-O-acetyl-4-dehydro-5,7,3,4-tetra-O-benzyl-(+)-catechin and (-)-epicatechin as a key building monomer. Tetrahedron Lett. 2008, 49, 3176-3180. [CrossRef]

22. Achilonu, M.C.; Bonnet, S.L.; van der Westhuizen, J.H. Synthesis of proanthocyanidins. Part 1. The first oxidative formation of the interflavanyl bond in procyanidins. Org. Lett. 2008, 10, 3865-3868. [CrossRef] [PubMed]

23. Mohri, Y.; Sagehashi, M.; Yamada, T.; Hattori, Y.; Morimura, K. An efficient synthesis of procyanidins using equimolar condensation of catechin and/or epicatechin catalyzed by ytterbium triflate. Heterocycles 2009, 79, 549-563. [CrossRef]

24. Watanabe, G.; Ohmori, K.; Suzuki, K. First regiocontrolled synthesis of procyanidin B6, a catechin dimer with rare connectivity: A halo-capping strategy for formation of 4,6-interflavan bonds. Chem. Commun. 2013, 49, 5210-5216. [CrossRef] [PubMed]

25. Saito, A.; Nakajima, N.; Tanaka, A.; Ubukata, M. Synthetic studies of proanthocyanidins. Highly stereoselective synthesis of catechin dimer, procyanidin-B3. Biosci. Biotechnol. Biochem. 2002, 66, 1764-1767. [CrossRef] [PubMed]

26. Saito, A.; Nakajima, N.; Tanaka, A.; Ubukata, M. Synthetic studies of proanthocyanidins. Part 2: Stereoselective gram-scale synthesis of procyanidin-B3. Tetrahedron 2002, 58, 7829-7837. [CrossRef]

27. Saito, A.; Nakajima, N.; Tanaka, A.; Ubukata, M. Synthetic studies of proanthocyanidins. Part 3: Stereoselective 3,4-cis catechin and catechin condensation by TMSOTf-catalyzed intramolecular coupling method. Tetrahedron Lett. 2003, 44, 5449-5452. [CrossRef] 
28. Saito, A.; Nakajima, N.; Tanaka, A.; Ubukata, M. Synthetic studies of proanthocyanidins. Part 4. The synthesis of procyanidin B1 and B4. TMSOTf-catalyzed cyclization of catechin and epicatechin condensation. Heterocycles 2003, 61, 287-298.

29. Saito, A.; Nakajima, N.; Matsuura, M.; Tanaka, A.; Ubukata, M. Synthetic studies of proanthocyanidins. Part 5. Highly stereoselective synthesis and inhibitory activity of Maillard reaction of 3,4-trans catechin and epicatechin dimers, procyanidin B1, B2, B3, B4 and their acetates. Heterocycles 2004, 62, 479-489.

30. Saito, A.; Tanaka, A.; Ubukata, M.; Nakajima, N. Efficient stereoselective synthesis of proanthocyanidin trimers with TMSOTf-catalyzed intermolecular condensation. Synlett 2004, 2014, 1069-1073. [CrossRef]

31. Saito, A.; Tanaka, A.; Ubukata, M.; Nakajima, N. Stereoselection of 3,4-cis and 3,4-trans catechin and catechin condensation under intramolecular coupling method. Synlett 2004, 2014, 2040-2042. [CrossRef]

32. Saito, A.; Doi, Y.; Matsuura, N.; Tanaka, A.; Ubukata, M.; Nakajima, N. Systematic synthesis of four epicatechin series procyanidin trimers and their inhibitory activity on the Maillard reaction and antioxidant activity. Bioorg. Med. Chem. 2004, 12, 4783-4790. [CrossRef] [PubMed]

33. Saito, A.; Enomoto, M.; Tanaka, A.; Doi, Y.; Shoji, K.; Mizushina, Y.; Ikawa, H.; Yoshida, H.; Matsuura, N.; Nakajima, N. Stereoselective synthesis of procyanidin B3-3-O-gallate and 3,3"-di-O-gallate, and their abilities as antioxidant and DNA polymerase inhibitor. Tetrahedron 2004, 60, 12043-12049. [CrossRef]

34. Saito, A.; Mizushina, Y.; Ikawa, H.; Yoshida, H.; Doi, Y.; Tanaka, A.; Nakajima, N. Systematic synthesis of galloyl-substituted procyanidin B1 and B2, and their ability of DPPH radical scavenging activity and inhibitory activity of DNA polymerases. Bioorg. Med. Chem. 2005, 13, 2759-2771. [CrossRef] [PubMed]

35. Sakuda, H.; Saito, A.; Mizushina, Y.; Ikawa, H.; Yoshida, H.; Nakajima, N. Synthesis of galloyl-substituted procyanidin B4 series, and their DPPH radical scavenging activity and DNA polymerase inhibitory activity. Heterocycles 2006, 67, 175-188.

36. Saito, A.; Mizushina, Y.; Tanaka, A.; Nakajima, N. Versatile synthesis of epicatechin series procyanidin oligomers, and their antioxidant and DNA polymerase inhibitory activity. Tetrahedron 2009, 65, 7422-7428. [CrossRef]

37. Ishihara, S.; Doi, S.; Harui, K.; Okamoto, T.; Okamoto, S. Development of a new synthetic strategy for procyanidin dimer condensation using peracetylated electrophiles. Heterocycles 2014, 88, 1595-1602.

38. Okamoto, S.; Ishihara, S.; Okamoto, T.; Doi, S.; Harui, K.; Higashino, Y.; Kawasaki, T.; Nakajima, N.; Saito, A. Inhibitory activity of synthesized acetylated procyanidin $\mathrm{B}_{1}$ analogues against HeLa S3 cells proliferation. Molecules 2014, 19, 1775-1785. [CrossRef] [PubMed]

39. Hamada, Y.; Takano, S.; Ayano, Y.; Tokunaga, M.; Koashi, T.; Hamada, M.; Nakajima, N.; Saito, A. Structure-Activity relationship of oligomeric flavan-3-ols: Importance of upper-unit B-ring hydroxyl groups in the dimeric structure for strong activities. Molecules 2015, 20, 18870-18885. [CrossRef] [PubMed]

40. Mori, K.; Ayano, Y.; Hamada, Y.; Hojima, T.; Tanaka, R.; Higashino, Y.; Izuno, M.; Okamoto, T.; Kawasaki, T.; Hamada, M.; Nakajima, N.; Saito, A. Role of 2,3-cis structure of (-)-epicatechin-3,5-O-digallate in inhibition of HeLa S3 cell proliferation. Nat. Prod. Chem. Res. 2015, 3, 172.

41. Flecher, A.C.; Porter, L.J.; Haslam, E.; Gupta, R.K. Plant proanthocyanidins. Part 3. Conformational and configurational studies of natural procyanidins. J. Chem. Soc. Perkin Trans. 1 1977, 14, 1628-1637. [CrossRef]

Sample Availability: Samples of the compounds $\mathbf{1}$ and $\mathbf{2}$ are available from the authors. 